\title{
Correlates of Krishi Dainandini Readers with their Farm Information Utilization Behaviour
}

\author{
P.T. Shinde, D.D. Suradkar and M.B. Shinde* \\ Department of Extension Education, College of Agriculture, Latur, Vasantrao Naik \\ Marathwada Krishi Vidyapeeth, Parbhani-431402 (M.S.), India \\ *Corresponding author
}

\section{A B S T R A C T}

The study was conducted in Latur and Beed districts of Marathwada region. Population for the study comprised of respondents who were readers of krishi

\section{Keywords}

Farm information utilization behaviour, Krishi Dainandini reders

Article Info

Accepted:

04 June 2019

Available Online:

10 July 2019 dainandini in Latur and Beed districts. From Latur district Latur, Ausa and from Beed district Ambejogai and Kaij tahsils were selected randomly. Respondents were selected randomly from the list of Krishi Dainandini readers which is collected from ATMA office of Latur and Beed districts. The total sample size was one hundred and twenty respondents which who are readers of Krishi Dainadini readers. One shot case study method of ex-post-facto research design was adopted for this study. Data was coded, tabulated, analysed and interpreted using suitable statistical parameters. The results showed that most of the readers (68.34\%) had medium farm information utilization behaviour and independent variables like education, land holding, annual income, social participation, reading habit, economic motivation and cosmopoliteness had positive correlation with farm information utilization behaviour of the respondents at 5 per cent and 1 per cent significant level respectively. Age and occupation were negative and nonsignificant correlation with farm information utilization behaviour.

\section{Introduction}

The annual farm publications under different titles are being published every year by all the State Agricultural Universities of Maharashtra. The Krishi Dainandini, an annual farm publication is being published by Vasantrao Naik Marathwada Krishi Vidyapeeth, Parbhani. Every year Krishi Dainandini is made available to farmers, offices of State Department of Agriculture, Libraries, Colleges etc Krishi Dainandini is most credible source of farm information. It contains variety of farm information related to farming such as new crop production technologies like improved varieties, cultivation practices, plant protection and post-harvest technology. It also provides information on agro based subsidiary occupations like dairy, sheep and goat rearing, 
apiaries, sericulture, vermi-compost, IPM, organic farming etc. The Krishi Dainandini provide technological know-how to the farmers at regular intervals and also increase the knowledge of farmers regarding improved package of practices of different crops and other allied fields.

\section{Materials and Methods}

The study was conducted in Latur and beed districts of Marathwada region. Population for the study comprised of respondents who were readers of krishi dainandini in Latur and Beed districts. From latur district Latur, Ausa and from beed district Ambejogai and kaij tahsils were selected randomly. Respondents were selected randomly from the list of Krishi Dainandini readers which is collected from ATMA office of Latur and Beed districts.

The total sample size was one hundred and twenty respondents which who are readers of Krishi Dainadini readers. One shot case study method of ex-post-facto research design was adopted for this study. The schedule of farm information utilization behaviour was developed with help of teacher to quantify farm information utilization behaviour was measured by computing the score. Final score was calculated by combining all scores and made as category low (upto 11), medium (12 to 19$)$ and high comfortable (20 \& above).

\section{Results and Discussion}

\section{Profile characteristics of the Krishi Dainandini readers}

The first objective of the study was to describe profile of Krishi Dainandini readers. Majority of the respondents belong to middle Age group. Majority of the respondents were educated up to higher secondary school level. Most of the respondents 37.50 per cent were having semi medium i.e. 2.01 to 4.00 ha land holding. More than half of the respondents 51.66 per cent engaged in agriculture. Majority of the respondents 7.33 per cent were in medium annual income. Majority 61.66 per cent of respondents were from medium social participation group followed by low social participation group and high social participation group. Majority of the respondents 66.66 per cent were medium reading habit (Table 1$)$.

\section{Farm information utilization behaviour}

The result showed that forty topics towards the farm information utilization behaviour of Krishi Dainandini readers. In that most of the readers 66.66 per cent were full and 4.17 per cent were partially farm information utilization behaviour about the pest management. 58.33 per cent of the respondents were full farm information utilization behaviour about Organic and chemical fertilizers. 54.17 per cent and 18.33 per cent of the readers were full and partially farm utilization behaviour respectively about integrated nutrient management. 51.67 per cent and 15.00 per cent readers were full and partially farm information utilization behaviour about respectively to use of biofertilizers whereas 47.50 per cent and 16.67 per cent of the readers were full and partially farm information utilization behaviour about sugarcane cultivation. 45.83 per cent of the respondents were full farm information utilization behaviour about disease management. Only 06.67 per cent, 05.00 per cent, 04.16 per cent and 03.33 per cent readers full farm information utilization behaviour about the agriculture engineering, home science, agriculture forestry, biogas and biodiesel respectively.

The data regarding to level of farm information utilization are given in table 2. 
Table.1 Profile characteristics of Krishi Dainadini readers

\begin{tabular}{|c|c|c|c|}
\hline Profile characters & Category & Frequency & Percentage \\
\hline \multirow{3}{*}{ Age } & Young age (Up to 31 year) & 24 & 20.00 \\
\hline & Middle age (between 31 to 47 year) & 75 & 62.50 \\
\hline & Old age (Above 47 year) & 21 & 17.50 \\
\hline \multirow[t]{10}{*}{ Education } & Illiterate & 00 & 00.00 \\
\hline & Only read and write & 12 & 10.00 \\
\hline & Primary education (Std $1^{\text {st }}$ to $\left.4^{\text {th }}\right)$ & 29 & 24.17 \\
\hline & Secondary education $\left(\operatorname{Std} 5^{\text {th }}\right.$ to $\left.10^{\text {th }}\right)$ & 29 & 24.17 \\
\hline & Higher secondary education $\left(11^{\text {th }}\right.$ to $\left.12^{\text {th }}\right)$ & 40 & 33.33 \\
\hline & Diploma & 03 & 02.50 \\
\hline & Graduated & 04 & 03.33 \\
\hline & Post Graduate & 03 & 02.50 \\
\hline & Ph.D. & 00 & 00.00 \\
\hline & Other & 00 & 00.00 \\
\hline \multirow[t]{5}{*}{ Land holding } & Marginal (up to $1 \mathrm{ha}$ ) & 23 & 19.17 \\
\hline & Small (1.01 to 2.00$)$ & 42 & 35.00 \\
\hline & Semi-medium (2.01 to 4.00$)$ & 45 & 37.50 \\
\hline & Medium (4.01 to 10.00$)$ & 10 & 08.33 \\
\hline & Big (10.01 and above) & 00 & 00.00 \\
\hline \multirow[t]{5}{*}{ Occupation } & Farm labour & 14 & 11.66 \\
\hline & Caste occupation & 20 & 16.66 \\
\hline & Business & 17 & 14.16 \\
\hline & Agriculture & 62 & 51.66 \\
\hline & Service & 7 & 05.83 \\
\hline \multirow[t]{3}{*}{ Annual income } & Low (Up to Rs. $1,20,290)$ & 22 & 18.33 \\
\hline & Medium (Rs. $1,20,291$ to $3,12,209$ ) & 88 & 73.33 \\
\hline & High (Rs.3,12,210 and above) & 10 & 08.34 \\
\hline \multirow[t]{3}{*}{ Social participation } & Low (Up to 6) & 28 & 23.33 \\
\hline & $\operatorname{Medium(7~to~} 12)$ & 74 & 61.66 \\
\hline & High (13 and above) & 18 & 15.00 \\
\hline \multirow[t]{3}{*}{ Reading habit } & Poor (Up to 4) & 26 & 21.67 \\
\hline & Medium (5 to 6 ) & 80 & 66.66 \\
\hline & Good (7 and above) & 14 & 11.67 \\
\hline \multirow{3}{*}{$\begin{array}{l}\text { Economic } \\
\text { motivation }\end{array}$} & Low (Up to 13) & 19 & 15.83 \\
\hline & Medium (14 to 16$)$ & 85 & 70.83 \\
\hline & High (17 and above) & 16 & 13.34 \\
\hline \multirow[t]{3}{*}{ Cosmopoliteness } & Low (Up to 1$)$ & 15 & 12.50 \\
\hline & Medium (2 to 3 ) & 85 & 70.83 \\
\hline & High (4 and above) & 20 & 16.67 \\
\hline
\end{tabular}


Table.2 Distribution of readers according to their specific farm information utilization behaviour

\begin{tabular}{|c|c|c|c|c|}
\hline \multirow[t]{2}{*}{ Sl. No. } & \multirow[t]{2}{*}{ Name of Topics } & \multicolumn{3}{|c|}{ Farm information utilization behaviour } \\
\hline & & Full & Partially & Not at all \\
\hline 1) & Research recommendation & $\begin{array}{c}28 \\
(23.33)\end{array}$ & $\begin{array}{c}17 \\
(14.17)\end{array}$ & $\begin{array}{c}75 \\
(62.50)\end{array}$ \\
\hline 2) & Extention eduction & $\begin{array}{c}22 \\
(18.33)\end{array}$ & $\begin{array}{c}10 \\
(08.33)\end{array}$ & $\begin{array}{c}88 \\
(73.34)\end{array}$ \\
\hline 3) & Agriculture economics & $\begin{array}{c}13 \\
(10.83)\end{array}$ & $\begin{array}{c}12 \\
(10.00)\end{array}$ & $\begin{array}{c}95 \\
(79.16)\end{array}$ \\
\hline 4) & $\begin{array}{l}\text { Protection of crop varieties } \\
\text { and farmer rights }\end{array}$ & $\begin{array}{c}13 \\
(10.83)\end{array}$ & $\begin{array}{c}8 \\
(06.67)\end{array}$ & $\begin{array}{c}99 \\
(82.50)\end{array}$ \\
\hline 5) & Agriculture climate & $\begin{array}{c}26 \\
(21.67)\end{array}$ & $\begin{array}{c}18 \\
(15.00)\end{array}$ & $\begin{array}{c}76 \\
(63.63)\end{array}$ \\
\hline 6) & Selection of soil & $\begin{array}{c}40 \\
(33.33)\end{array}$ & $\begin{array}{c}17 \\
(14.17)\end{array}$ & $\begin{array}{c}63 \\
(52.50)\end{array}$ \\
\hline 7) & Soil testing & $\begin{array}{c}47 \\
(39.17)\end{array}$ & $\begin{array}{c}22 \\
(18.33)\end{array}$ & $\begin{array}{c}51 \\
(42.50)\end{array}$ \\
\hline 8) & $\begin{array}{l}\text { Organic and chemical } \\
\text { fertilizers }\end{array}$ & $\begin{array}{c}70 \\
(58.33)\end{array}$ & $\begin{array}{c}25 \\
(20.83)\end{array}$ & $\begin{array}{c}25 \\
(20.83)\end{array}$ \\
\hline 9) & $\begin{array}{l}\text { Integrated nutrient } \\
\text { management }\end{array}$ & $\begin{array}{c}65 \\
(54.17)\end{array}$ & $\begin{array}{c}22 \\
(18.33)\end{array}$ & $\begin{array}{c}33 \\
(27.50)\end{array}$ \\
\hline 10) & Use of biofertilizers & $\begin{array}{c}62 \\
(51.67)\end{array}$ & $\begin{array}{c}18 \\
(15.00)\end{array}$ & $\begin{array}{c}40 \\
(33.33)\end{array}$ \\
\hline 11) & Use of vermicompost & $\begin{array}{c}50 \\
(41.67)\end{array}$ & $\begin{array}{c}25 \\
(20.83)\end{array}$ & $\begin{array}{c}45 \\
(37.50)\end{array}$ \\
\hline 12) & Organic farming & $\begin{array}{c}30 \\
(25.00)\end{array}$ & $\begin{array}{c}19 \\
(15.83)\end{array}$ & $\begin{array}{c}71 \\
(59.17)\end{array}$ \\
\hline 13) & land farming management & $\begin{array}{c}17 \\
(14.17)\end{array}$ & $\begin{array}{c}12 \\
(10.00)\end{array}$ & $\begin{array}{c}91 \\
(75.83)\end{array}$ \\
\hline 14) & $\begin{array}{l}\text { Crop production and } \\
\text { management }\end{array}$ & $\begin{array}{c}28 \\
(23.33)\end{array}$ & $\begin{array}{c}15 \\
(12.50)\end{array}$ & $\begin{array}{c}77 \\
(64.17)\end{array}$ \\
\hline 15) & Water management & $\begin{array}{c}20 \\
(16.67)\end{array}$ & $\begin{array}{c}15 \\
(12.50)\end{array}$ & $\begin{array}{c}85 \\
(70.83)\end{array}$ \\
\hline 16) & $\begin{array}{l}\text { Plant biotechnology and } \\
\text { tissue culture }\end{array}$ & $\begin{array}{c}20 \\
(16.67)\end{array}$ & $\begin{array}{c}10 \\
(08.33)\end{array}$ & $\begin{array}{c}90 \\
(75.00)\end{array}$ \\
\hline 17) & Crop improved varieties & $\begin{array}{c}35 \\
(29.17)\end{array}$ & $\begin{array}{c}12 \\
(10.00)\end{array}$ & $\begin{array}{c}73 \\
(60.83)\end{array}$ \\
\hline 18) & Crop cultivation method & $\begin{array}{c}45 \\
(37.50)\end{array}$ & $\begin{array}{c}15 \\
(12.50)\end{array}$ & $\begin{array}{c}60 \\
(50.00)\end{array}$ \\
\hline 19) & Sugarcane cultivation & $\begin{array}{c}57 \\
(47.50)\end{array}$ & $\begin{array}{c}20 \\
(16.67)\end{array}$ & $\begin{array}{c}43 \\
(35.83)\end{array}$ \\
\hline
\end{tabular}




\begin{tabular}{|c|c|c|c|c|}
\hline 20) & Fodder crop & $\begin{array}{c}15 \\
(12.50)\end{array}$ & $\begin{array}{c}12 \\
(10.00)\end{array}$ & $\begin{array}{c}93 \\
(77.50)\end{array}$ \\
\hline 21) & Weed management & $\begin{array}{c}20 \\
(16.67)\end{array}$ & $\begin{array}{c}25 \\
(20.83)\end{array}$ & $\begin{array}{c}75 \\
(62.50)\end{array}$ \\
\hline 22) & Pest management & $\begin{array}{c}80 \\
(66.66)\end{array}$ & $\begin{array}{c}5 \\
(4.17)\end{array}$ & $\begin{array}{c}35 \\
(29.17)\end{array}$ \\
\hline 23) & Cultivation of fruits & $\begin{array}{c}27 \\
(22.50)\end{array}$ & $\begin{array}{c}16 \\
(13.33)\end{array}$ & $\begin{array}{c}77 \\
(64.17)\end{array}$ \\
\hline 24) & Vegetable cultivation & $\begin{array}{c}30 \\
(25.00)\end{array}$ & $\begin{array}{c}10 \\
(08.33)\end{array}$ & $\begin{array}{c}80 \\
(66 . .67)\end{array}$ \\
\hline 25) & Floriculture & $\begin{array}{c}15 \\
(12.50)\end{array}$ & $\begin{array}{c}14 \\
(11.67)\end{array}$ & $\begin{array}{c}91 \\
(75.83)\end{array}$ \\
\hline 26) & Green house technology & $\begin{array}{c}19 \\
(15.83)\end{array}$ & $\begin{array}{c}18 \\
(15.00)\end{array}$ & $\begin{array}{c}83 \\
(69.17)\end{array}$ \\
\hline 27) & Agriculture forestry & $\begin{array}{c}5 \\
(04.16)\end{array}$ & $\begin{array}{c}3 \\
(02.50)\end{array}$ & $\begin{array}{c}112 \\
(93.33)\end{array}$ \\
\hline 28) & Disease management & $\begin{array}{c}55 \\
(45.83)\end{array}$ & $\begin{array}{c}16 \\
(13.33)\end{array}$ & $\begin{array}{c}49 \\
(65.84)\end{array}$ \\
\hline 29) & Cow nutrition and health & $\begin{array}{c}48 \\
(40.00)\end{array}$ & $\begin{array}{c}19 \\
(15.83)\end{array}$ & $\begin{array}{c}53 \\
(44.17)\end{array}$ \\
\hline 30) & Buffalo nutrition and health & $\begin{array}{c}10 \\
(08.33)\end{array}$ & $\begin{array}{c}12 \\
(10.00)\end{array}$ & $\begin{array}{c}98 \\
(81.67)\end{array}$ \\
\hline 31) & Goat nutrition and health & $\begin{array}{c}24 \\
(20.00)\end{array}$ & $\begin{array}{c}11 \\
(09.17)\end{array}$ & $\begin{array}{c}85 \\
(70.83)\end{array}$ \\
\hline 32) & Poultry nutrition and health & $\begin{array}{c}18 \\
(15.00)\end{array}$ & $\begin{array}{c}7 \\
(05.83)\end{array}$ & $\begin{array}{c}95 \\
(79.17)\end{array}$ \\
\hline 33) & Agriculture Engineering & $\begin{array}{c}8 \\
(06.67)\end{array}$ & $\begin{array}{c}9 \\
(07.50)\end{array}$ & $\begin{array}{c}103 \\
(85.83)\end{array}$ \\
\hline 34) & Value added products & $\begin{array}{c}15 \\
(12.50)\end{array}$ & $\begin{array}{c}10 \\
(08.33)\end{array}$ & $\begin{array}{c}95 \\
(79.17)\end{array}$ \\
\hline 35) & Sericulture & $\begin{array}{c}20 \\
(16.67)\end{array}$ & $\begin{array}{c}11 \\
(09.17)\end{array}$ & $\begin{array}{c}89 \\
(74.16)\end{array}$ \\
\hline 36) & Mushroom cultivation & $\begin{array}{c}00 \\
(00.00)\end{array}$ & $\begin{array}{c}4 \\
(03.33)\end{array}$ & $\begin{array}{c}116 \\
(96.67)\end{array}$ \\
\hline 37) & Biogas and biodiesel & $\begin{array}{c}4 \\
(03.33)\end{array}$ & $\begin{array}{c}12 \\
(10.00)\end{array}$ & $\begin{array}{c}104 \\
(11.67)\end{array}$ \\
\hline 38) & Fishery & $\begin{array}{c}00 \\
(00.00)\end{array}$ & $\begin{array}{c}5 \\
(04.17)\end{array}$ & $\begin{array}{c}115 \\
(95.83)\end{array}$ \\
\hline 39) & $\begin{array}{l}\text { Improved } \\
\text { equipments }\end{array}$ & $\begin{array}{c}48 \\
(40.00)\end{array}$ & $\begin{array}{c}20 \\
(16.67)\end{array}$ & $\begin{array}{c}52 \\
(43.33)\end{array}$ \\
\hline 40) & Home science technology & $\begin{array}{c}6 \\
(05.00)\end{array}$ & $\begin{array}{c}12 \\
(10.00)\end{array}$ & $\begin{array}{c}102 \\
(85.00)\end{array}$ \\
\hline
\end{tabular}

Overall farm information utilization behaviour 
Table.3 Distribution of reader farmers of Krishi Darshani according to overall level of farm information utilization behaviour

\begin{tabular}{|c|c|c|c|}
\hline Sl. No. & $\begin{array}{l}\text { Overall level of farm information } \\
\text { utilization behaviour }\end{array}$ & Frequency & Percentage \\
\hline 1. & Low (Up to 11$)$ & 17 & 14.16 \\
\hline 2. & Medium (12 to 19$)$ & 82 & 68.34 \\
\hline \multirow[t]{2}{*}{3.} & High (20 and above) & 21 & 17.50 \\
\hline & Total & 120 & 100.00 \\
\hline
\end{tabular}

Table.4 Relationship between profile characteristics of the readers and their farm information utilization behaviour

\begin{tabular}{|l|l|c|}
\hline Sl. No. & Variables & Correlation Coefficient (r) \\
\hline $\mathbf{1 .}$ & Age & $-0.102^{\mathrm{NS}}$ \\
\hline $\mathbf{2 .}$ & Education & $0.197^{*}$ \\
\hline $\mathbf{3 .}$ & Land holding & $0.275^{* *}$ \\
\hline $\mathbf{4 .}$ & Occupation & $-0.002^{\mathrm{NS}}$ \\
\hline $\mathbf{5 .}$ & Annual Income & $0.263^{* *}$ \\
\hline $\mathbf{6 .}$ & Social Participation & $0.291^{* *}$ \\
\hline $\mathbf{7 .}$ & Reading habit & $0.328^{* *}$ \\
\hline $\mathbf{8 .}$ & Economic Motivation & $0.259^{* *}$ \\
\hline $\mathbf{9 .}$ & Cosmopoliteness & $0.217^{*}$ \\
\hline
\end{tabular}

* Significant at 0.05 level of probability.

** Significant at 0.01 level of probability.

NS- Non significant

The data from table 3 show that, readers of Krishi Dainandini had medium farm information utilization behaviour (68.34 per cent), followed by 17.50 per cent of reader farmers had high farm information utilization behaviour while 14.16 per cent of reader farmers had low farm information utilization behaviour.

These findings are consistent with Garudkar (2010).

Relationship between the profile characteristics and farm information utilization behaviour

The results revealed that out of nine independent variables, seven variables had exhibited positive and significant relationship with farm information utilization behaviour of readers. Education, land holding, annual income, social participation, reading habit, economic motivation and cosmopoliteness, had positive correlation with farm information utilization behaviour of the respondents at 5 per cent and 1 per cent significant level respectively. Age and occupation were negative and non-significant correlation with farm information utilization behaviour (Table $4)$.

It was concluded that, majorities of the respondents were middle age group, higher secondary school level of education, semi medium land holding, more than half respondents engaged in agriculture and medium annual income category. Further it could observe that majority of the 
respondents, medium level of social participation, reading habit, economic motivation and cosmopoliteness. Also clearly observed that majority of the respondents had a medium farm information utilization behaviour. Therefore, it is suggested that to improve the quality of the Krishi Dainandini by considering the suggestions expressed by the readers. Success stories or experience features of progressive farmers may be included in Krishi Dainandini, so as to motivate other reader farmers. Thus there is still scope to improve the Krishi Dainandini to reach high utilization behaviour.

\section{References}

Chavan, V.U., (2002). Study of readership and effectiveness of Shetibhati magazine as perceived by the readers. Unpublished M.Sc. (Agri.). Thesis,
MAU, Parbhani (M.S.).

Garudkar, S.B., (2010). Krishi Darshani Utilization Behaviour of Farmers in College Development B-lock, Pune. M.Sc. (Agri.) Thesis, M.P.K.V., Rahuri.

Hanumanaikar, R.H., Manjunath and, L. and Natikar, K.V., (2012). An analytical study on reading habits of Kannada farm magazine readers. Karnataka $J$. Agric. Sci., 25 (4): 441-445.

Kate, S.M., (1992). Evaluation of farm magazine ShriSugi published by M.P.K.V., Rahuri at farmers level. Unpublished M.Sc. (Agri.).Thesis, M.P.K.V., Rahuri.

Shinde M.R., (2016). Utility perception of VNMKV calender by the readers. M.Sc. (Agri.) Thesis, MAU, Parbhani.

\section{How to cite this article:}

Shinde, P.T., D.D. Suradkar and Shinde, M.B. 2019. Correlates of Krishi Dainandini Readers with their Farm Information Utilization Behaviour. Int.J.Curr.Microbiol.App.Sci. 8(07): 180186. doi: https://doi.org/10.20546/ijcmas.2019.807.023 